\title{
Decreasing Energy Costs in Epitaxial Growth from Gas Phase System by Optimization of Pressure
}

\author{
E.L. Pankratov ${ }^{1, *}$, E.A. Bulaeva ${ }^{2}$ \\ ${ }^{1}$ Nizhny Novgorod State University, 23 Gagarin avenue, Nizhny Novgorod, 603950, Russia \\ ${ }^{2}$ Nizhny Novgorod State University of Architecture and Civil Engineering, 65 Il'insky street, Nizhny Novgorod, 603950, Russia \\ *Corresponding Author: elp2004@mail.ru,
}

Copyright (C) 2013 Horizon Research Publishing All rights reserved.

\begin{abstract}
In this paper we introduce an approach to decrease energy costs during growth an epitaxial layers from gas phase by minimization velocity of flow of composition of gases (gas-carrier and materials in gas phase, which are used for formation epitaxial layer) after leaving epitaxial growth from gas phase system. As a result of minimization of the velocity one can obtain minimization of pressure of compressor and decreasing of energy cost of the epitaxial growth.
\end{abstract}

Keywords Epitaxial Growth From Gas Phase; Decreasing Energy Costs; Optimization Of Pressure

\section{Introduction}

Problem of growth of epitaxial structures with required properties is one of actual problems to improvement of properties, which has been manufactured based on heterostructures [1-8]. To growth heterostructures one can use different ways [1-3]. One way to growth heterostructures is using growth of epitaxial layers from gas phase [1]. Main aim of the present work is choosing such conditions of growth, which correspond to decreasing of energy cost during growth of layers of heterostructure.

\section{Statement of the Problem}

Let us consider epitaxial growth from gas phase system in neighborhood of reaction zone (see Fig. 1). Within the epitaxial growth system a disk with a substrate is rotating with angular velocity $\omega$. An epitaxial layer will be grown on the substrate. Materials, which are used for growth the epitaxial layer, are carried to the zone of reaction by gas-carrier under the pressure $P$ $(z)$ and velocity of flow of composition $\vec{v}$. The velocity has three components with account cylindrical system of coordinates $v_{r}, v_{\varphi}$ and $v_{z}$. Main aim of the present work is choosing of minimal pressure in initial part of reaction zone $P(-L)$ under condition, that pressure in final part of reaction zone is equal to atmospheric one $P(L)=P_{a t m}$. In this case dependence of pressure in area of reaction zone on coordinate $z$ could be approximated as $P(z)=0,5\left(P_{\text {atm }}+P_{\text {in }}\right)+\left(P_{\text {atm }}-P_{i n}\right) z / 2 L$.

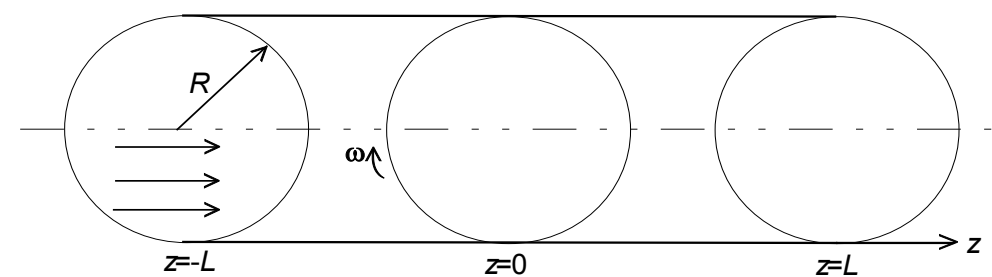

Figure 1. Epitaxial growth from gas phase system in area of reaction zone

\section{Method of Solution}

Framework the paper we consider, that radius of disk $R$ is essentially larger, than thickness of diffusive and boundary 
layers. We also consider gas course as laminar. Balance equation for concentration of deposit material during convection mass transport could be written as

$$
\frac{\partial C(r, \varphi, z, t)}{\partial t}=\operatorname{div}\{D \cdot \operatorname{grad}[C(r, \varphi, z, t)]-\vec{v}(r, \varphi, z, t) \cdot C(r, \varphi, z, t)\},
$$

where $D$ is the gas diffusion coefficient, $\vec{v}$ is the velocity of flow. The first term of the Eq.(1) describes diffusion of gas from beginning of reactor $(z=-L)$ to end of the reactor $(z=L)$. The second term of the Eq. (1) describes convection transport of gas with velocity of flow $\vec{v}$ under influence of external pressure $P$.

Let us consider the regime of the limiting flow, when all forthcoming to the disk molecules of deposit material are deposing on the substrate. In this case boundary and initial conditions could be written as

$$
\begin{gathered}
C(r, \varphi,-L, t)=C_{0}(t), C(r, \varphi, 0, t)=0, C(r, 0, z, t)=C(r, 2 \pi, z, t), C(r, \varphi, z, 0)=C_{0}(t) \delta(z+L), \\
C(0, \varphi, z, t) \neq \infty,\left.\frac{\partial C(r, \varphi, z, t)}{\partial r}\right|_{r=R}=0,\left.\frac{\partial C(r, \varphi, z, t)}{\partial \varphi}\right|_{\varphi=0}=\left.\frac{\partial C(r, \varphi, z, t)}{\partial \varphi}\right|_{\varphi=2 \pi} .
\end{gathered}
$$

The conditions describe absents of gas flow through casing of reactor at $r=R$, absence of infinite increasing of approximation of concentration of gas at $r=0$, conditions of periodicity of concentration of gas with varying of angular coordinate $\varphi$, initial condition and condition at $z=-L$ correspond to presents of concentration of gas on beginning of reactor in initial moment of time, reason of considered value of concentration in the end of reaction $z=L$ is necessity of minimization of expenditure of materials, which are used for growth of heterostructure.

The Eq.(1) including into itself unknown velocity of flow $\vec{v}$. We determine the velocity by solution the Navier-Stokes equation

$$
\frac{\partial \vec{v}}{\partial t}+(\vec{v} \cdot \nabla) \vec{v}=-\nabla\left(\frac{P}{\rho}\right)+v \Delta \vec{v},
$$

where $P$ is the pressure of compressor, $\rho$ is the density of gas, $v$ is the kinematical viscosity. One can find total acceleration of gas in the left side of the Eq.(3). The first term of the right side of the equation describes moving of gas under influence of external force (pressure of compressor). The second term of the right side of the Eq. (3) describes deceleration of moving of gas under influence of viscosity.

Equations for components of velocity framework cylindrical system of coordinates could be written as

$$
\begin{gathered}
\frac{\partial v_{r}}{\partial t}+v_{r} \frac{\partial v_{r}}{\partial r}+\frac{v_{\varphi}}{r} \frac{\partial v_{r}}{\partial \varphi}+v_{z} \frac{\partial v_{r}}{\partial z}=v\left(\frac{\partial^{2} v_{r}}{\partial r^{2}}+\frac{\partial^{2} v_{r}}{\partial r \partial z}-\frac{\partial^{2} v_{r}}{\partial z^{2}}+\frac{\partial^{2} v_{z}}{\partial r \partial z}\right)-\frac{\partial}{\partial r}\left(\frac{P}{\rho}\right) \\
\frac{\partial v_{\varphi}}{\partial t}+v_{r} \frac{\partial v_{\varphi}}{\partial r}+\frac{v_{\varphi}}{r} \frac{\partial v_{\varphi}}{\partial \varphi}+v_{z} \frac{\partial v_{\varphi}}{\partial z}=v\left(\frac{1}{r} \frac{\partial^{2} v_{r}}{\partial r \partial \varphi}+\frac{2}{r^{2}} \frac{\partial^{2} v_{\varphi}}{\partial \varphi^{2}}-\frac{1}{r^{2}} \frac{\partial^{2} v_{r}}{\partial \varphi \partial}+\frac{\partial^{2} v_{\varphi}}{\partial z^{2}}\right)-\frac{1}{r} \frac{\partial}{\partial \varphi}\left(\frac{P}{\rho}\right) \\
\frac{\partial v_{z}}{\partial t}+v_{r} \frac{\partial v_{z}}{\partial r}+\frac{v_{\varphi}}{r} \frac{\partial v_{z}}{\partial \varphi}+v_{z} \frac{\partial v_{z}}{\partial z}=v\left(\frac{\partial^{2} v_{r}}{\partial z^{2}}+\frac{\partial^{2} v_{z}}{\partial r^{2}}+\frac{1}{r^{2}} \frac{\partial^{2} v_{z}}{\partial \varphi^{2}}\right)-\frac{\partial}{\partial z}\left(\frac{P}{\rho}\right) .
\end{gathered}
$$

Boundary and initial conditions for Eqs.(4) by using "adhesion" conditions on the surface of disk, homogeneity and one-dimensionality of flow in initial part of reaction zone could be written as

$$
\begin{aligned}
& v_{r}(r, \varphi,-L, t)=0, v_{r}(r, \varphi, 0, t)=0, v_{r}(r, \varphi, L, t)=0, v_{r}(r, 0, z, t)=v_{r}(r, 2 \pi, z, t), v_{r}(0, \varphi, z, t) \neq \infty, v_{\varphi}(r, \varphi, 0, t)=\omega r, v_{\varphi}(r, \varphi,-L, t)=0, v_{\varphi}(r, \varphi, L, t)=0, \\
& \left.\frac{\partial v_{r}(r, \varphi, z, t)}{\partial r}\right|_{r=0}=\left.\frac{\partial v_{r}(r, \varphi, z, t)}{\partial r}\right|_{r=R}=0,\left.\frac{\partial v_{\varphi}(r, \varphi, z, t)}{\partial \varphi}\right|_{\varphi=0}=\left.\frac{\partial v_{\varphi}(r, \varphi, z, t)}{\partial \varphi}\right|_{\varphi=2 \pi}, v_{\varphi}(r, 0, z, t)=v_{\varphi}(r, 2 \pi, z, t), v_{\varphi}(0, \varphi, z, t) \neq \infty,(5)
\end{aligned}
$$

$v_{z}(r, \varphi,-L, t)=V_{0}, v_{z}(r, \varphi, 0, t)=0, v_{z}(r, \varphi, L, t)=0, v_{z}(r, 0, z, t)=v_{z}(r, 2 \pi, z, t), v_{z}(0, \varphi, z, t) \neq \infty, v_{r}(r, \varphi, z, 0)=0, v_{\varphi}(r, \varphi, z, 0)=0, v_{z}(r, \varphi,-L, t)=V_{0}$.

The conditions describe absents of gas flow through casing of reactor at $r=R$, absence of infinite increasing of approximation of velocity of gas at $r=0$, conditions of periodicity of velocity of flow with varying of angular coordinate $\varphi$, initial condition and condition at $z=-L$ correspond to presents of gas on beginning of reactor in initial moment of time, reason of considered value of velocity in the end of reaction $z=L$ is necessity of minimization of energy cost during growth of a heterostructure. 
Let us transform the Eqs.(4) to the following form

$$
\begin{gathered}
\frac{\partial v_{r}}{\partial t}=v\left(\frac{\partial^{2} v_{r}}{\partial r^{2}}+\frac{\partial^{2} v_{r}}{\partial r \partial z}-\frac{\partial^{2} v_{r}}{\partial z^{2}}+\frac{\partial^{2} v_{z}}{\partial r \partial z}\right)-\frac{\partial}{\partial r}\left(\frac{P}{\rho}\right)-v_{r} \frac{\partial v_{r}}{\partial r}-\frac{v_{\varphi}}{r} \frac{\partial v_{r}}{\partial \varphi}-v_{z} \frac{\partial v_{r}}{\partial z} \\
\frac{\partial v_{\varphi}}{\partial t}=v\left(\frac{1}{r} \frac{\partial^{2} v_{r}}{\partial r \partial \varphi}+\frac{2}{r^{2}} \frac{\partial^{2} v_{\varphi}}{\partial \varphi^{2}}-\frac{1}{r^{2}} \frac{\partial^{2} v_{r}}{\partial \varphi \partial z}+\frac{\partial^{2} v_{\varphi}}{\partial z^{2}}\right)-\frac{1}{r} \frac{\partial}{\partial \varphi}\left(\frac{P}{\rho}\right)-v_{r} \frac{\partial v_{\varphi}}{\partial r}-\frac{v_{\varphi}}{r} \frac{\partial v_{\varphi}}{\partial \varphi}-v_{z} \frac{\partial v_{\varphi}}{\partial z} \\
\frac{\partial v_{z}}{\partial t}=v\left(\frac{\partial^{2} v_{r}}{\partial z^{2}}+\frac{\partial^{2} v_{z}}{\partial r^{2}}+\frac{1}{r^{2}} \frac{\partial^{2} v_{z}}{\partial \varphi^{2}}\right)-\frac{\partial}{\partial z}\left(\frac{P}{\rho}\right)-v_{r} \frac{\partial v_{z}}{\partial r}-\frac{v_{\varphi}}{r} \frac{\partial v_{z}}{\partial \varphi}-v_{z} \frac{\partial v_{z}}{\partial z} .
\end{gathered}
$$

We determine solution of the above system of equations by method of averaging of function correction [9-11]. Framework the approach we determine the first-order approximations of components of velocity of flow by replacement the components in right sides of the Eqs.(4d)-(4f) on their not yet known average values $v_{r} \rightarrow \alpha_{1 r}, v_{\varphi} \rightarrow \alpha_{1 \varphi}, v_{z} \rightarrow \alpha_{1 z}$. Relations for the first-order approximations of components could be written as

$$
\frac{\partial v_{1 r}}{\partial t}=-\frac{\partial}{\partial r}\left(\frac{P}{\rho}\right), \frac{\partial v_{1 \varphi}}{\partial t}=-\frac{1}{r} \frac{\partial}{\partial \varphi}\left(\frac{P}{\rho}\right), \frac{\partial v_{1 z}}{\partial t}=-\frac{\partial}{\partial z}\left(\frac{P}{\rho}\right) .
$$

We determine the average values $\alpha_{1 r}, \alpha_{1 \varphi}, \alpha_{1 z}$ by using the following standard relations

$$
\alpha_{1 r}=\int_{0}^{\Theta} \int_{0}^{R} r \int_{0}^{2 \pi} \int_{-L}^{L} v_{1 r} d w d v d u d \tau, \alpha_{1 \varphi}=\int_{0}^{\Theta} \int_{0}^{R} r \int_{0}^{2 \pi} \int_{-L}^{L} v_{1 \varphi} d w d v d u d \tau, \alpha_{1 z}=\int_{0}^{\Theta} \int_{0}^{R} r \int_{0}^{2 \pi} \int_{-L}^{L} v_{1 z} d w d v d u d \tau
$$

where $\Theta$ is the continuance of taking of the flow. However in this situation the first-order approximations of components of velocity of flow independent from the average values. Final relations for the approximations could be obtain by integration of all relations of (5) on time. The final form could be written as

$$
v_{1 r}=-\frac{\partial}{\partial r} \int_{0}^{t} \frac{P}{\rho} d \tau, v_{1 \varphi}=-\frac{1}{r} \frac{\partial}{\partial \varphi} \int_{0}^{t} \frac{P}{\rho} d \tau, v_{1 z}=-\frac{\partial}{\partial z} \int_{0}^{t} \frac{P}{\rho} d \tau .
$$

The second-order approximations of components of velocity of flow could be calculated by replacement of the required components in the right sides of Eqs.(4d)-(4f) on the following sums $v_{r} \rightarrow \alpha_{2 r}+v_{1 r}, v_{\varphi} \rightarrow \alpha_{2 \varphi}+v_{1 \varphi}, v_{z} \rightarrow \alpha_{2 z}+v_{1 z}$. The approximations could be written as

$$
\begin{gathered}
\frac{\partial v_{2 r}}{\partial t}=v\left(\frac{\partial^{2} v_{1 r}}{\partial r^{2}}+\frac{\partial^{2} v_{1 r}}{\partial r \partial z}-\frac{\partial^{2} v_{1 r}}{\partial z^{2}}+\frac{\partial^{2} v_{1 z}}{\partial r \partial z}\right)-\frac{\partial}{\partial r}\left(\frac{P}{\rho}\right)- \\
-\left(\alpha_{2 r}+v_{1 r}\right) \frac{\partial v_{1 r}}{\partial r}-\frac{\left(\alpha_{2 \varphi}+v_{1 \varphi}\right)}{r} \frac{\partial v_{1 r}}{\partial \varphi}-\left(\alpha_{2 z}+v_{1 z}\right) \frac{\partial v_{1 r}}{\partial z} \\
\frac{\partial v_{2 \varphi}}{\partial t}=v\left(\frac{1}{r} \frac{\partial^{2} v_{1 r}}{\partial r \partial \varphi}+\frac{2}{r^{2}} \frac{\partial^{2} v_{1 \varphi}}{\partial \varphi^{2}}-\frac{1}{r^{2}} \frac{\partial^{2} v_{1 r}}{\partial \varphi \partial z}+\frac{\partial^{2} v_{1 \varphi}}{\partial z^{2}}\right)-\frac{1}{r} \frac{\partial}{\partial \varphi}\left(\frac{P}{\rho}\right)- \\
-\left(\alpha_{2 r}+v_{1 r}\right) \frac{\partial v_{1 \varphi}}{\partial r}-\frac{\left(\alpha_{2 \varphi}+v_{1 \varphi}\right)}{r} \frac{\partial v_{1 \varphi}}{\partial \varphi}-\left(\alpha_{2 z}+v_{1 z}\right) \frac{\partial v_{1 \varphi}}{\partial z} \\
\frac{\partial v_{2 z}}{\partial t}=v\left(\frac{\partial^{2} v_{1 r}}{\partial z^{2}}+\frac{\partial^{2} v_{1 z}}{\partial r^{2}}+\frac{1}{r^{2}} \frac{\partial^{2} v_{1 z}}{\partial \varphi^{2}}\right)-\frac{\partial}{\partial z}\left(\frac{P}{\rho}\right)- \\
-\left(\alpha_{2 r}+v_{1 r}\right) \frac{\partial v_{1 z}}{\partial r}-\frac{\left(\alpha_{2 \varphi}+v_{1 \varphi}\right)}{r} \frac{\partial v_{1 z}}{\partial \varphi}-\left(\alpha_{2 z}+v_{1 z}\right) \frac{\partial v_{1 z}}{\partial z}
\end{gathered}
$$

Integration on time of the above equations leads to final relations of the second-order approximations of components of velocity of flow 


$$
\begin{gathered}
v_{2 r}=v \int_{0}^{t}\left(\frac{\partial^{2} v_{1 r}}{\partial r^{2}}+\frac{\partial^{2} v_{1 r}}{\partial r \partial z}-\frac{\partial^{2} v_{1 r}}{\partial z^{2}}+\frac{\partial^{2} v_{1 z}}{\partial r \partial z}\right) d \tau-\frac{\partial}{\partial r}\left(\int_{0}^{t} \frac{P}{\rho} d \tau\right)- \\
-\int_{0}^{t}\left(\alpha_{2 r}+v_{1 r}\right) \frac{\partial v_{1 r}}{\partial r} d \tau-\int_{0}^{t} \frac{\left(\alpha_{2 \varphi}+v_{1 \varphi}\right)}{r} \frac{\partial v_{1 r}}{\partial \varphi} d \tau-\int_{0}^{t}\left(\alpha_{2 z}+v_{1 z}\right) \frac{\partial v_{1 r}}{\partial z} d \tau \\
v_{2 \varphi}=v \int_{0}^{t}\left(\frac{1}{r} \frac{\partial^{2} v_{1 r}}{\partial r \partial \varphi}+\frac{2}{r^{2}} \frac{\partial^{2} v_{1 \varphi}}{\partial \varphi^{2}}-\frac{1}{r^{2}} \frac{\partial^{2} v_{1 r}}{\partial \varphi \partial}+\frac{\partial^{2} v_{1 \varphi}}{\partial z^{2}}\right) d \tau-\frac{1}{r} \frac{\partial}{\partial \varphi}\left(\int_{0}^{t} \frac{P}{\rho} d \tau\right)- \\
-\int_{0}^{t}\left(\alpha_{2 r}+v_{1 r}\right) \frac{\partial v_{1 \varphi}}{\partial r} d \tau-\int_{0}^{t} \frac{\left(\alpha_{2 \varphi}+v_{1 \varphi}\right)}{r} \frac{\partial v_{1 \varphi}}{\partial \varphi} d \tau-\int_{0}^{t}\left(\alpha_{2 z}+v_{1 z}\right) \frac{\partial v_{1 \varphi}}{\partial z} d \tau \\
-\int_{0}^{t}\left(\alpha_{2 r}+v_{1 r}\right) \frac{\partial v_{1 z}}{\partial r} d \tau-\int_{0}^{t} \frac{\partial^{2} v_{1 r}}{\partial z^{2}}+\frac{\partial^{2} v_{1 z}}{\left.\partial r^{2}+\frac{1}{r^{2}} \frac{\partial^{2} v_{1 z}}{\partial \varphi^{2}}\right) d \tau-\frac{\partial}{\partial z}\left(\int_{1 \varphi}^{t} \frac{P}{\rho} d \tau\right)-}\left(\alpha_{2 \varphi}+v_{1 \varphi}\right) \frac{\partial v_{1 z}}{\partial \varphi} d \tau-\int_{0}^{t}\left(\alpha_{2 z}+v_{1 z}\right) \frac{\partial v_{1 z}}{\partial z} d \tau
\end{gathered}
$$

We determine the average values $\alpha_{2 r}, \alpha_{2 \varphi}, \alpha_{2 z}$ by using the standard relations

$$
\begin{gathered}
\alpha_{2 r}=\int_{0}^{\Theta} \int_{0}^{R} r \int_{0}^{2 \pi} \int_{-L}^{L}\left(v_{2 r}-v_{1 r}\right) d w d v d u d \tau, \alpha_{2 \varphi}=\int_{0}^{\Theta} \int_{0}^{R} r \int_{0}^{2 \pi} \int_{-L}^{L}\left(v_{2 \varphi}-v_{1 \varphi}\right) d w d v d u d \tau, \\
\alpha_{2 z}=\int_{0}^{\Theta} \int_{0}^{R} r \int_{0}^{2 \pi} \int_{-L}^{L}\left(v_{2 z}-v_{1 z}\right) d w d v d u d \tau .
\end{gathered}
$$

Substitution of the first- and the second-order approximations of components of velocity of flow in the relations (9) gives us possibility to obtain the following system of equations for the required average values $\alpha_{2 r}, \alpha_{2 \varphi}, \alpha_{2 z}$

$$
\left\{\begin{array}{l}
A_{1} \alpha_{2 r}+B_{1} \alpha_{2 \varphi}+C_{1} \alpha_{2 z}=D_{1} \\
A_{2} \alpha_{2 r}+B_{2} \alpha_{2 \varphi}+C_{2} \alpha_{2 z}=D_{2} \\
A_{3} \alpha_{2 r}+B_{3} \alpha_{2 \varphi}+C_{3} \alpha_{2 z}=D_{3}
\end{array}\right.
$$

where

$$
\begin{aligned}
& A_{1}=1+\int_{0}^{\Theta}(\Theta-t) \int_{0}^{R} r \int_{0}^{2 \pi} \int_{-L}^{L} \frac{\partial v_{1 r}}{\partial r} d w d v d u d \tau, B_{1}=\int_{0}^{\Theta}(\Theta-t) \int_{0}^{R} \int_{0}^{2 \pi} \int_{-L}^{L} \frac{\partial v_{1 r}}{\partial \varphi} d w d v d u d \tau, C_{1}=C_{2}=\frac{\pi}{2} \Theta^{2} R^{2} V_{0} \\
& D_{1}=v \int_{0}^{\Theta}(\Theta-t) \int_{0}^{R} r \int_{0}^{2 \pi} \int_{-L}^{L}\left(\frac{\partial^{2} v_{1 r}}{\partial r^{2}}+\frac{\partial^{2} v_{1 r}}{\partial r \partial z}-\frac{\partial^{2} v_{1 r}}{\partial z^{2}}+\frac{\partial^{2} v_{1 z}}{\partial r \partial z}\right) d w d v d u d \tau-\int_{0}^{\Theta} \int_{0}^{R} r \int_{0}^{2 \pi} \int_{-L}^{L} v_{1 r} \frac{\partial v_{1 r}}{\partial r} d w d v d u \times \\
& \times(\Theta-t) d \tau-\int_{0}^{\Theta}(\Theta-t) \int_{0}^{R} \int_{0}^{2 \pi} \int_{-L}^{L} v_{1 \varphi} \frac{\partial v_{1 r}}{\partial \varphi} d w d v d u d \tau-\frac{\pi}{8} \Theta^{2} R^{2} V_{0}^{2}, A_{2}=\int_{0}^{\Theta}(\Theta-t) \int_{0}^{R} r \int_{0}^{2 \pi} \int_{-L}^{L} \frac{\partial v_{1 r}}{\partial r} d w d v d u d \tau \\
& B_{2}=1+\int_{0}^{\Theta}(\Theta-t) \int_{0}^{R} \int_{0}^{2 \pi} \int_{-L}^{L} \frac{\partial v_{1 r}}{\partial \varphi} d w d v d u d \tau, D_{2}=v \int_{0}^{\Theta}(\Theta-t) \int_{0}^{R} r \int_{0}^{2 \pi} \int_{-L}^{L}\left(\frac{1}{r} \frac{\partial^{2} v_{1 r}}{\partial r \partial \varphi}+\frac{2}{r^{2}} \frac{\partial^{2} v_{1 \varphi}}{\partial \varphi^{2}}-\frac{1}{r^{2}} \frac{\partial^{2} v_{1 r}}{\partial \varphi \partial z}+\right. \\
& \left.+\frac{\partial^{2} v_{1 \varphi}}{\partial z^{2}}\right) d w d v d u d \tau-\int_{0}^{\Theta}(\Theta-t) \int_{0}^{R} r \int_{0}^{2 \pi} \int_{-L}^{L} v_{1 r} \frac{\partial v_{1 r}}{\partial r} d w d v d u d \tau-\int_{0}^{\Theta}(\Theta-t) \int_{0}^{R} \int_{0}^{2 \pi} \int_{-L}^{L} v_{1 \varphi} \frac{\partial v_{1 r}}{\partial \varphi} d w d v d u d \tau-
\end{aligned}
$$




$$
\begin{gathered}
-\frac{\pi}{8} \Theta^{2} R^{2} V_{0}^{2}, A_{3}=\int_{0}^{\Theta}(\Theta-t) \int_{0}^{R} r \int_{0}^{2 \pi} \int_{-L}^{L} \frac{\partial v_{1 z}}{\partial r} d w d v d u d \tau, B_{3}=\int_{0}^{\Theta}(\Theta-t) \int_{0}^{R} r \int_{0}^{2 \pi} \int_{-L}^{L} \frac{\partial v_{1 z}}{\partial \varphi} d w d v d u d \tau, \\
C_{3}=1+\frac{\pi}{2} \Theta^{2} R^{2} V_{0}, D_{3}=v \int_{0}^{\Theta}(\Theta-t) \int_{0}^{R} r \int_{0}^{2 \pi} \int_{-L}^{L}\left(\frac{\partial^{2} v_{1 r}}{\partial z^{2}}+\frac{\partial^{2} v_{1 z}}{\partial r^{2}}+\frac{1}{r^{2}} \frac{\partial^{2} v_{1 z}}{\partial \varphi^{2}}\right) d w d v d u d \tau-\int_{0}^{\Theta}(\Theta-t) \int_{0}^{R} r \times \\
\quad \times \int_{0}^{2 \pi} \int_{-L}^{L} v_{1 r} \frac{\partial v_{1 z}}{\partial r} d w d v d u d \tau-\int_{0}^{\Theta}(\Theta-t) \int_{0}^{R} r \int_{0}^{2 \pi} \int_{-L}^{L} v_{1 \varphi} \frac{\partial v_{1 z}}{\partial \varphi} d w d v d u d \tau-\frac{\pi}{8} \Theta^{2} R^{2} V_{0}^{2} .
\end{gathered}
$$

As the parameters $A_{i}, B_{i}$ and $C_{i}$ have been marked coefficients of equations to determine average values of the second-order approximations of components of velocity of flow $\alpha_{2 r}, \alpha_{2 \varphi}, \alpha_{2 z}$. This marking gives us possibility to write the system of equations (10) in shorter and more demonstrable form. The system of equations could be solved by standard approaches [12]. The result of calculation could be written as

$$
\alpha_{2 r}=\Delta_{r} / \Delta, \alpha_{2 \varphi}=\Delta_{\varphi} / \Delta, \alpha_{2 z}=\Delta_{z} / \Delta,
$$

where

$$
\begin{gathered}
\Delta=A_{1}\left(B_{2} C_{3}-B_{3} C_{2}\right)-B_{1}\left(A_{2} C_{3}-A_{3} C_{2}\right)+C_{1}\left(A_{2} B_{3}-A_{3} B_{2}\right), \Delta_{r}=D_{1}\left(B_{2} C_{3}-B_{3} C_{2}\right)-B_{1}\left(D_{2} C_{3}-D_{3} C_{2}\right)+ \\
+C_{1}\left(D_{2} B_{3}-D_{3} B_{2}\right), \Delta_{\varphi}=D_{1}\left(B_{2} C_{3}-B_{3} C_{2}\right)-B_{1}\left(D_{2} C_{3}-D_{3} C_{2}\right)+C_{1}\left(D_{2} B_{3}-D_{3} B_{2}\right), \Delta_{z}=A_{1}\left(B_{2} D_{3}-B_{3} D_{2}\right)- \\
-B_{1}\left(A_{2} D_{3}-A_{3} D_{2}\right)+D_{1}\left(A_{2} B_{3}-A_{3} B_{2}\right) .
\end{gathered}
$$

In this section we calculated components of velocity of flow of materials in gas phase, which are used for growth an epitaxial layer, and gas-carrier in the second-order approximation framework the method of averaging of function corrections. Usually the second-order approximation is enough good approximation to make qualitative analysis and to obtain some quantitative results.

\section{Discussion}

Based on relations, calculated in the previous section, we estimate minimal value of pressure in initial part of reaction zone $P(-L)=P_{i n}$. The obtained value of pressure has been obtained based on condition of equality of output velocity to zero. Choosing of zero value of output velocity of gas flow gives us possibility to obtain maximal decreasing of energy costs during epitaxial growth. Usually value of required pressure in initial part of reaction zone $P_{i n}$ is equal to few (depends on parameters) atmospheric pressures $P_{a t m}$.

\section{Conclusion}

In this work we introduce an approach to decrease energy cost of growth of epitaxial layers from gas phase. At the same time we introduce an analytical approach to model of velocities of flow during growth of an epitaxial growth.

\section{Acknowledgments}

This work is supported by the contract 11.G34.31.0066 of the Russian Federation Government, grant of Scientific School of Russia and educational fellowship for scientific research.

\section{REFERENCES}

[1] I.P. Stepanenko. Basis of microelectronics. Moscow, Soviet radio (in Russian), 1980.

[2] V.G. Gusev, Yu.M. Gusev. Electronics. Moscow, Higher School (in Russian), 1991. 
[3] V.I. Lachin, N.S. Savelov. Electronics. Rostov-on-Don, Phenics, 2001.

[4] A.A. Vorob'ev, V.V. Korabl'ev, S.Yu. Karpov. Semiconductors. Vol. 37 (7). P. 98 (2003).

[5] L.M. Sorokin, N.V. Veselov, M.P. Shcheglov, A.E. Kalmykov, A.A. Sitnikova, N.A. Feoktistov, A.V. Osipov, S.A. Kukushkin. Technical Physics Letters. Vol. 34 (22). P. 68 (2008).

[6] V.V. Lundin, A.V. Sakharov, E.E. Zavarin, M.A. Sinitsin, A.E. Nikolaev, G.A. Mikhailovsky, P.N. Brunkov, V.V. Goncharov, B.Ya. Ber, D.Yu. Kazantsev, A.F. Tsatsul'nikov. Semiconductors. Vol. 43 (7). P. 996 (2009).

[7] Y. Li, L.E. Antonuk, Y. El-Mohri, Q. Zhao, H. Du, A. Sawant, Yi Wang. J. Appl. Phys. Vol. 99 (6). P. 064501 (2006).

[8] A. Chakraborty, H. Xing, M.D. Craven, S. Keller, T. Mates, J. S. Speck, S.P. Den Baars, U.K. Mishra. J. Appl. Phys. Vol. 96 (8). P. 4494 (2004).

[9] Yu.D. Sokolov. Applied Mechanics. Vol.1 (1). P. 23-35 (1955).

[10] E.L. Pankratov. J. Comp. Theor. Nanoscience. Vol.9 (1). P. 41-49 (2012).

[11] E.L. Pankratov, E.A. Bulaeva. Nanoscience and Nanotechnology Letters. Vol.5 (3). P. 418-426 (2013).

[12] G. Korn, T. Korn. Mathematical Handbook for scientists and engineers. Definitions, theorems and formulas for reference and review. Second edition. McGraw-Hill Book Company. New York, 1968. 